\title{
In Vivo Neutralization of P-Selectin Protects Feline Heart and Endothelium in Myocardial Ischemia and Reperfusion Injury
}

\author{
Andrew S. Weyrich, Xin-liang Ma, David J. Lefer, * Kurt H. Albertine, and Allan 'M. Lefer \\ Department of Physiology, Jefferson Medical College, Thomas Jefferson University, Philadelphia, Pennsylvania 19107-6799; and \\ *Division of Cardiology, Johns Hopkins University, School of Medicine, Baltimore, Maryland 21224
}

\begin{abstract}
The cardioprotective effects of an $\mathbf{m A b}$ to $\mathbf{P}$-selectin designated mAb PB1.3 was examined in a feline model of myocardial ischemia (MI) and reperfusion. PB1.3 (1 mg/ kg), administered after $80 \mathrm{~min}$ of ischemia (i.e., $10 \mathrm{~min}$ before reperfusion), significantly attenuated myocardial necrosis compared to a nonblocking mAb (NBP1.6) for P-selectin (15 \pm 3 vs $35 \pm 3 \%$ of area at risk, $P<0.01$ ). Moreover, endothelial release of endothelium derived relaxing factor, as assessed by relaxation to acetylcholine, was also significantly preserved in ischemic-reperfused coronary arteries isolated from cats treated with $\mathbf{m A b}$ PB1.3 compared to $\mathrm{mAb}$ NBP1.6 (67 \pm 6 vs $11 \pm 3, P<0.01)$. This endothelial preservation was directly related to reduced endothelial adherence of PMNs in ischemic-reperfused coronary arteries. Immunohistochemical localization of P-selectin was significantly upregulated in the cytoplasm of endothelial cells that lined coronary arteries and veins after $90 \mathrm{~min}$ of ischemia and $20 \mathrm{~min}$ of reperfusion. The principal site of intracytoplasmic expression was in venous vessels. mAb PB1.3 significantly decreased $(P<0.01)$ adherence of unstimulated PMNs to thrombin and histamine stimulated endothelial cells in a concentration-dependent manner in vitro. These results demonstrate that PMN adherence to endothelium by $\mathbf{P}$-selectin is an important early consequence of reperfusion injury, and a specific monoclonal antibody to $\mathbf{P}$-selectin exerts significant endothelial preservation and cardioprotection in myocardial ischemia and reperfusion. (J. Clin. Invest. 1993. 91:2620-2629.) Key words: myocardial necrosis $\bullet$ neutrophil adherence $\bullet$ endothelial dysfunction • coronary artery $\bullet \mathbf{P}$-selectin
\end{abstract}

\section{Introduction}

The proximity of the endothelium to circulating leukocytes makes it an important early target for neutrophil adherence and subsequent PMN-mediated damage (1). This adhesive interaction can be rapidly induced and is characterized by the expression of adhesion molecules such as P-selectin on the endothelial surface (2). P-selectin, also known as granule membrane protein-140, is an integral membrane glycoprotein located in platelets and in Weibel-Palade bodies of endothelial

Address correspondence to Allan M. Lefer, Ph.D., Department of Physiology, Jefferson Medical College, Thomas Jefferson University, 1020 Locust St., Philadelphia, PA 19107-6799.

Received for publication 4 September 1992 and in revised form 11 December 1992.

J. Clin. Invest.

(c) The American Society for Clinical Investigation, Inc

$0021-9738 / 93 / 06 / 2620 / 10 \quad \$ 2.00$

Volume 91, June 1993, 2620-2629 cells (3). Upon activation of the endothelium with agonists such as thrombin and histamine, the Weibel-Palade bodies fuse with the plasma membrane, and P-selectin is rapidly translocated to the endothelial cell surface in $\sim 5 \mathrm{~min}$, where it tethers circulating PMNs and positions them for activation by endothelial-bound platelet activating factor $(3,4)$.

This adhesive interaction between PMNs and endothelial cells is an early and requisite event in inflammatory disorders such as ischemia reperfusion injury $(5,6)$. Ischemia followed by reperfusion is also associated with a decrease in agonist-stimulated (7) and basal nitric oxide (NO) (8) release, which has also been shown to increase PMN adherence to endothelial cells (9). Moreover, this burst of free radical generation at the onset of reperfusion coincides with a marked reduction in endothelium-derived relaxing factor $(E D R F)^{1}(7)$ and precedes the induction of P-selectin expression on endothelial cells (10,11). Thus, rapid expression of P-selectin on endothelial cells may be responsible, at least in part, for the initial cascade of PMN-mediated effects that ultimately results in myocardial tissue injury after ischemia reperfusion. However, in vivo studies have not addressed the role of $\mathrm{P}$-selectin in regulating $\mathrm{PMN}$ recruitment in an ischemic-reperfusion setting. Therefore, the purposes of this study were $(a)$ to determine the effect of a monoclonal antibody to P-selectin on myocardial tissue injury associated with ischemia and reperfusion in cats; $(b)$ to relate the myocardial injury to endothelial dysfunction and adherence of PMNs to the coronary vascular endothelium; and $(c)$ to examine P-selectin expression by endothelial cells after ischemia and reperfusion and determine how this relates to PMN-endothelial interactions associated with reperfusion injury.

\section{Methods}

Monoclonal antibodies. mAbs PB1.3 (also known as Cy-1747) and NBP1.6 are murine $\mathrm{IgG}_{1}$ monoclonal antibodies raised against human P-selectin, which were obtained from Cytel Corp. (San Diego, CA). PB1.3 blocks the interaction between P-selectin and the sialyl Lewis X ligand while NBP1.6 does not block the interaction between P-selectin and the sialyl Lewis $\mathrm{X}$ ligand although it recognizes P-selectin. The complete characterization of these antibodies is previously described (12).

Experimental protocol. Adult male cats $(2.6-3.4 \mathrm{~kg})$ were anesthetized intravenously with sodium pentobarbital $(30 \mathrm{mg} / \mathrm{kg})$. An intratracheal cannula was inserted through a midline incision, and all cats were placed on intermittent positive-pressure ventilation (small ani-

1. Abbreviations used in this paper: ACh, acetylcholine; ECG, electrocardiogram; EDRF, endothelium-derived relaxing factor; HR, heart rate; ICAM, intercellular adhesion molecule; $\mathrm{K}-\mathrm{H}$, Krebs-Henseleit; LAD, left anterior descending (coronary artery); LCX, left circumflex; MABP, mean arterial blood pressure; MI, myocardial ischemia; NO, nitric oxide; PAF, platelet-activating factor; PRI, pressure-rate index; PVC, premature ventricular contraction; $R$, reperfusion. 
mal respirator; Harvard Apparatus, Dover, MA). A polyethylene catheter was inserted into the right external jugular vein for supplementary pentobarbital infusion to maintain a surgical plane of anesthesia and for administration of antibodies. An additional polyethylene catheter was inserted through the left femoral artery and positioned in the abdominal aorta for measurement of mean arterial blood pressure (MABP) via a pressure transducer (Statham P23AC; Gould, Inc., Cleveland, $\mathrm{OH}$ ). After a midsternal thoracotomy, the pericardium was incised and a 3-0 silk ligature was placed around the left anterior descending (LAD) coronary artery $8-10 \mathrm{~mm}$ from its origin. Standard lead II of the scalar electrocardiogram (ECG) was used to determine heart rate (HR) and ST segment elevation. ST segment elevations were determined by analysis of an ECG recording at $50 \mathrm{~mm} / \mathrm{s}$ every $20 \mathrm{~min}$. The ECG and MABP were continuously recorded on an oscillographic recorder (model 7; Grass Instrument Co., Quincy, MA), and the pressure-rate index (PRI), an approximation of myocardial oxygen demand, was calculated as the product of MABP and HR divided by 1,000 .

After completing all surgical procedures, the cats were allowed to stabilize for $30 \mathrm{~min}$, at which time baseline readings of ECG and MABP were recorded. Myocardial ischemia (MI) was produced by tightening the previously placed reversible ligature around the LAD to completely occlude the vessel. This was designated as time $0.80 \mathrm{~min}$ after coronary occlusion (i.e., 10 min before reperfusion [R]), $1 \mathrm{mg} /$ $\mathrm{kg}$ of mAb PB1.3 (i.e., specific mAb for P-selectin [Cytel, San Diego, $\mathrm{CA}]$ ) or mAb NBP1.6 (i.e., a nonblocking antibody for P-selectin [Cytel]) was given intravenously as a bolus. $10 \mathrm{~min}$ later, the LAD ligature was untied and the ischemic myocardium was reperfused for $270 \mathrm{~min}$. The animals were randomly divided into two major groups of six cats each consisting of $\mathrm{MI}+\mathrm{R}$ cats receiving either $\mathrm{mAb} \mathrm{PB} 1.3$ or $\mathrm{mAb}$ NBP1.6. In addition, one additional group consisting of three sham MI $+R$ cats receiving PB1.3 (1 mg/kg) was also included in this study. Sham MI + R cats were subjected to the same surgical procedures as $\mathrm{MI}+\mathrm{R}$ cats, except that the LAD coronary artery was not occluded.

Myocardial tissue analysis. At the end of the 270-min postreperfusion period, the ligature around the LAD was retightened. $20 \mathrm{ml}$ of $0.5 \%$ Evans blue was injected over a period of $1 \mathrm{~min}$ into the left ventricle to stain the area of myocardium that was perfused by the patent coronary arteries. The area at risk was thus determined by negative staining. Immediately after this injection, the heart was rapidly excised and placed in warmed, oxygenated Krebs-Henseleit (K-H) buffer consisting of (in $\mathrm{mM}$ ): $\mathrm{NaCl}, 118 ; \mathrm{KCl}, 4.75 ; \mathrm{CaCl}_{2} \cdot 2 \mathrm{H}_{2} \mathrm{O}, 2.54$; $\mathrm{KH}_{2} \mathrm{PO}_{4}, 1.19 ; \mathrm{MgSO}_{4} \cdot 7 \mathrm{H}_{2} \mathrm{O}, 1.19 ; \mathrm{NaHCO}_{3}, 12.5$, and glucose, 10.0 . The left circumflex (LCX) and the LAD coronary arteries were isolated and removed for subsequent study of coronary ring vasoactivity. The right ventricle and great vessels were then carefully removed, and the left ventricle was sliced parallel to the atrioventricular groove in 3-mm thick sections according to previously reported methods (7). The unstained portion of the myocardium (i.e., the total area at risk) was separated from the Evans blue-stained portion of the myocardium (i.e., the area not at risk). The area at risk was again sectioned into 1 -mm thick slices and incubated in $0.1 \%$ nitroblue tetrazolium in phosphate buffer at $\mathrm{pH} 7.4$ and $37^{\circ} \mathrm{C}$ for $15 \mathrm{~min}$. The tetrazolium dye forms a blue formazan complex in the presence of coenzymes and dehydrogenases. The irreversibly injured or necrotic portion of the myocardium at risk that did not stain was separated from the stained portion of the myocardium (i.e., the ischemic but nonnecrotic area). The nonischemic, ischemic nonnecrotic, and ischemic necrotic tissues were subsequently weighed and the results were expressed as the area at risk indexed to the total left ventricular mass, the area of necrotic tissue indexed to the area at risk, and the area of necrotic tissue indexed to the total left ventricular mass. In three additional cats receiving only $0.9 \%$ $\mathrm{NaCl}$ as a vehicle, the above procedures were repeated, except that half of the area at risk was incubated with $20 \mu \mathrm{g} / \mathrm{ml}$ of mAb PB1.3 to rule out the possibility that $\mathrm{mAb} P B 1.3$ altered the staining properties of the nitroblue tetrazolium. The area of necrotic tissue expressed as a percentage of the area at risk was $38.9 \pm 1.0 \%$ in the control area at risk samples and $39.6 \pm 1.8 \%$ in the area at risk samples incubated with $\mathrm{mAb}$ PB1.3.
These values are not significantly different indicating that $\mathrm{mAb}$ PB1.3 had no effect on nitroblue tetrazolium staining properties.

Isolated coronary ring studies. Both LAD and LCX coronary segments were removed and placed into warmed $\mathrm{K}-\mathrm{H}$ buffer. Isolated coronary vessels were cleaned and cut into rings $2-3 \mathrm{~mm}$ in length. The rings were then mounted on stainless steel hooks, suspended in 10-ml tissue baths, and connected to force displacement transducers (FT-03; Grass Instrument $\mathrm{Co}$.) to record changes in force on an oscillographic recorder (model 7; Grass Instrument $\mathrm{Co}$.). The baths were filled with $10 \mathrm{ml}$ of $\mathrm{K}-\mathrm{H}$ buffer and aerated at $37^{\circ} \mathrm{C}$ with a gas mixture of $95 \% \mathrm{O}_{2}$ and $5 \% \mathrm{CO}_{2}$. Coronary rings were initially stretched to give a preload of $0.5 \mathrm{~g}$ of force and equilibrated for $60-90 \mathrm{~min}$. During this period, the $\mathrm{K}-\mathrm{H}$ buffer in the tissue baths was replaced every $15 \mathrm{~min}$. After equilibration, the rings were then exposed to $100 \mathrm{nM}$ U-46619 (9,11-epoxymethano- $\mathrm{PGH}_{2}$; Biomol Research Laboratories, Plymouth Meeting, PA), a thromboxane $A_{2}$ mimetic, to generate about $0.5 \mathrm{~g}$ of developed force. Once a stable contraction was obtained, acetylcholine (ACh), an endothelium-dependent vasodilator, was added to the bath in cumulative concentrations of $0.1,1,10$, and $100 \mathrm{nM}$. After the response stabilized, the rings were washed and allowed to equilibrate to baseline once again. The procedure was repeated with another endothelium-dependent vasodilator, A-23187, $(1,10,100$, and $1,000 \mathrm{nM})$, and then again with an endothelium-independent vasodilator, acidified $\mathrm{NaNO}_{2},(0.1$, $1,10$, and $100 \mu \mathrm{M})$, at $\mathrm{pH} 2$.

Cat PMN isolation and labeling. In six $\mathrm{MI}+\mathrm{R}$ cats, peripheral blood $(10 \mathrm{ml})$ was collected from the femoral artery before the onset of ischemia reperfusion and anticoagulated with citrate-phosphate-dextrose solution (Sigma Immunochemicals, St. Louis, MO) (1.4:10 vol/ vol anticoagulant to whole blood). $20 \mathrm{ml}$ of $0.9 \% \mathrm{NaCl}$ was immediately infused to replace the blood volume and the cats were allowed to stabilize for $30 \mathrm{~min}$. The blood was placed into round-bottom polycarbonate centrifuge tubes (Nalge, Rochester, NY) and PMNs were isolated by a procedure modified from Lafredo and Olsen (13). Plateletrich plasma (PRP) was obtained by centrifuging blood at $400 \mathrm{~g}$ for 20 min (Econospin tabletop swinging bucket centrifuge; Sorvall Instruments, Wilmington, DE). PRP was decanted and centrifuged at 2,500 $g$ for $10 \mathrm{~min}$ to obtain platelet-poor plasma (PPP). PPP was then mixed with isotonic Percoll (Sigma Immunochemicals) (9 vol Percoll/ $1 \mathrm{vol} 1.5 \mathrm{M} \mathrm{NaCl}$ ) to produce Percoll-PPP density gradients of 80,62 , and $50 \% .8 \mathrm{ml}$ of $6 \%$ dextran (average mol wt 60,000-90,000, Sigma, St. Louis, MO) and $20 \mathrm{ml}$ of PBS were added to the erythrocyte-leukocyte pellet from the initial $400 \mathrm{~g}$ centrifugation. After mixture by inversion, the erythrocytes were allowed to settle for 40-60 $\mathrm{min}$. The leukocyte enriched fraction was resuspended in $1 \mathrm{ml}$ of $0.9 \% \mathrm{NaCl}$ and layered onto the Percoll /PPP gradient. The suspensions were then centrifuged at $1,000 \mathrm{~g}$ for $40 \mathrm{~min}$ at $4^{\circ} \mathrm{C}$ in a refrigerated centrifuge (RC2B; Sorvall Instruments, Newtown, CT). PMNs were collected from the 62-82\% interface and washed twice with PBS before being assayed for viability using trypan blue exclusion. PMN preparations obtained by this method were typically $>95 \%$ pure and $>95 \%$ viable.

Isolated autologously, PMNs were then labeled with a fluorescent dye (PKH2; Zynaxis, Malvern, PA) according to the method of Yuan and Fleming (14). One $\mathrm{ml}$ of the diluent was added to a loose cell pellet containing $\sim 10$ million cells. $1 \mathrm{ml}$ of PKH2-GL dye $(4 \mathrm{mM})$ was added to the cell suspension and then mixed for $5 \mathrm{~min}$ by inversion. 2 $\mathrm{ml}$ of PBS (containing 10\% PPP in PBS) was added to stop the reaction and another $5 \mathrm{ml}$ of PBS was added to the suspension. Cells were then centrifuged at $400 \mathrm{~g}$ for $10 \mathrm{~min}$ at room temperature. The supernatant was removed, and the cells were resuspended in PBS and then recounted. This labeling procedure yields cat PMNs possessing normal morphology and function (15).

Effect of in vivo administration of $M A b P B 1.3$ on PMN adherence to ischemic-reperfused coronary artery endothelium. During the PMN isolation and labeling period, the cats were subjected to $90 \mathrm{~min}$ of ischemia and $270 \mathrm{~min}$ of reperfusion (three cats received $\mathrm{mAb}$ PB1.3 and three cats received mAb NBP1.6) and the ischemic-reperfused LAD and control LCX coronary segments were isolated and removed as previously described (15). The arteries were opened and placed into 
the cell culture dishes containing $3 \mathrm{ml}$ of $\mathrm{K}-\mathrm{H}$ buffer. Autologously labeled PMNs $(400,000 \mathrm{PMNs} / \mathrm{ml})$ were then added to the arteries and allowed to incubate for $20 \mathrm{~min}$ in a metabolic shaker bath at $37^{\circ} \mathrm{C}$. The arteries were removed and PMN adherence to coronary artery endothelium was counted using fluorescence microscopy as previously described (8). The number of PMNs were counted in duplicate and reported as PMN per square millimeter of the coronary endothelial surface.

Effect of mAb PB1.3 on unstimulated PMN adherence to stimulated coronary artery endothelium. In six additional cats, peripheral blood $(80 \mathrm{ml})$ was collected from the femoral artery and anticoagulated with citrate-phosphate-dextrose solution. PMNs were isolated as described above. Hearts from each control cat were removed and placed in warm oxygenated K-H buffer. Both the LAD and LCX coronary arteries were removed and cut in 2-3-mm segments. The arteries were then opened, and placed into a cell culture dish filled with $3 \mathrm{ml}$ of K-H buffer. To stimulate endothelial cells, the coronary rings were incubated for 10 min with either $2 \mathrm{U} / \mathrm{ml}$ of thrombin (Sigma Immunochemicals) or 10 $\mu \mathrm{M}$ histamine (Sigma Immunochemicals). After this $10 \mathrm{~min}$ incubation period, the coronary ring segments were removed and placed in fresh K-H solution. In the first series, labeled PMNs (400,000 PMNs/ $\mathrm{ml}$ ) were added to thrombin-stimulated endothelial cells alone and in combination with increasing concentrations of mAb PB1.3 (5, 10, 20, 30 , and $40 \mu \mathrm{g} / \mathrm{ml}$ ). In the second series, labeled PMNs were added concomitantly with $20 \mu \mathrm{g} / \mathrm{ml}$ of mAb PB1.3 to endothelial cells stimulated with either thrombin or histamine. After $20 \mathrm{~min}$, the coronary rings were removed and adherent PMNs were counted using fluorescence microscopy as described above.

Effect of $m A b P B 1.3$ on unstimulated PMN adherence to ischemicreperfused coronary artery endothelium. In three additional cats that were subjected to $90 \mathrm{~min}$ of ischemia and $20 \mathrm{~min}$ of reperfusion, ischemic-reperfused LAD and nonischemic LCX coronary artery segments were removed and prepared for PMN adherence studies as previously described. The rings were placed in the cell culture dishes and unstimulated PMNs (400,000 PMNs/ml) were added simultaneously with either $20 \mu \mathrm{g} / \mathrm{ml}$ of mAb PB1.3 or mAb NBP1.6 for $20 \mathrm{~min}$ in a metabolic shaker bath at $37^{\circ} \mathrm{C}$. Coronary artery segments were then removed from the culture dishes and PMN adherence to the endothelium was assessed as described above.

Immunohistochemical localization of P-selectin. To assess the cross-reactivity of mAb PB1.3 with feline endothelium, three cats were exposed to $90 \mathrm{~min}$ of ischemia followed by $20 \mathrm{~min}$ of reperfusion, and two cats were exposed to $90 \mathrm{~min}$ of ischemia followed by $270 \mathrm{~min}$ of reperfusion. The hearts were subsequently removed and the aorta was immediately cannulated and perfused with $\mathrm{K}-\mathrm{H}$ solution for $2 \mathrm{~min}$ at $50 \mathrm{mmHg}$. After this 2 min washout period, the $\mathrm{K}-\mathrm{H}$ solution was switched to $4 \%$ paraformaldehyde in PBS and the hearts were perfusion fixed for $5 \mathrm{~min}$. Full thickness slices of the left ventricular wall ( $1 \mathrm{~mm}$ in thickness and $5 \mathrm{~mm}$ in width) were fixed for $2 \mathrm{~h}$ at $4^{\circ} \mathrm{C}$. Slices, cut from the ischemic-reperfused region and the nonischemic region, were dehydrated in a graded series of acetone at $4^{\circ} \mathrm{C}$ and embedded (Immunobed; Polysciences Inc., Warrington, PA) at $4^{\circ} \mathrm{C}$ for $12 \mathrm{~h} .5-\mu \mathrm{m}$ thick sections were cut using glass knives and transferred to coated slides (Vectabond; Vector Laboratories, Burlingame, CA).

Immunohistochemical procedures on plastic sections were performed in the following sequence using a modification of the methods described previously by Beckstead et al. (16) using the avidin-biotin immunoperoxidase technique (Vectastain ABC Reagent; Vector Laboratories). Incubation of the primary antibody was carried out overnight at room temperature at dilutions of $1: 100,1: 1,000$, and 1:2,000 in PBS. Control preparations for immunohistochemistry included omission of the primary antibody and also incubation with the primary antibody but with omission of the secondary antibody (biotinylated IgG). The sections were examined using a Zeiss microscope.

Flow cytometric analysis of binding of PB1.3 to cat platelets. The binding of the mAb PB1.3 to freshly isolated cat platelets was determined by flow cytometric analysis. Briefly, peripheral arterial blood was collected from the femoral artery of four pentobarbital-anesthe- tized cats and was anticoagulated with $3.8 \%$ sodium citrate. The blood was placed into round-bottom polycarbonate centrifuge tubes and PRP was obtained by centrifuging the blood at $400 \mathrm{~g}$ for $20 \mathrm{~min}$. The PRP was removed and recentrifuged at $2,500 \mathrm{~g}$ to form a platelet pellet. The platelet pellet was washed twice in calcium-free Tyrode's solution containing $1 \%$ BSA. Platelets were resuspended in Dulbecco's PBS and $0.2 \%$ BSA and aliquots of $2 \times 10^{8}$ platelets $/ \mathrm{ml}$ were activated with human thrombin (Sigma Immunochemicals) at $0.25 \mathrm{U} / \mathrm{ml}$ for $20 \mathrm{~min}$ at room temperature in the presence of $4 \mathrm{mM} \mathrm{Ca}^{2+}$. After the thrombin incubation period, EDTA was added to the cell suspensions at a final concentration of $10 \mathrm{mM}$, and the cell suspensions were centrifuged (Eppendorf microcentrifuge; Brinkman Instruments, Westbury, NY) for $30 \mathrm{~s}$ at 7,000 rpm, and the supernatant was discarded. The platelets were then washed in PBS containing $10 \mathrm{mM}$ EDTA and centrifuged. Additional aliquots of platelets were treated in an identical manner with the exception of the thrombin treatment, and these platelet suspensions served as controls for these experiments. The platelet suspensions were treated with human block IgG $(4.0 \mathrm{mg} / \mathrm{ml}$ ) (Sigma Immunochemicals), and then PB1.3 (40 $\mu \mathrm{g} / \mathrm{ml}$ ) or human $\mathrm{IgG}_{1}$ (Coulter, Hialeah, FL) $(40 \mu \mathrm{g} / \mathrm{ml})$ was added to the cat platelets. The platelets were incubated at room temperature for $30 \mathrm{~min}$. The platelets were then washed in Dulbecco's PBS to remove any excess of the primary antibodies. $F\left(a b^{\prime}\right) 2$ fragments of a goat anti-mouse IgG-phycoerythrin conjugate (Tago, Inc., Burlingame, CA) was used as the secondary antibody reagent at a 1:100 dilution and the cells were placed on ice for $30 \mathrm{~min}$. The stained platelets were then washed and fixed in $1.0 \%$ paraformaldehyde and then analyzed on a flow cytometer (EPICS; Coulter, Hialeah, FL).

Platelet aggregation. Aggregation studies were performed on cat platelets that were washed as previously described. Platelets were aggregated with $0.1 \mathrm{U} / \mathrm{ml}$ of thrombin in the presence or absence of PB1.3 $(20-40 \mu \mathrm{g} / \mathrm{ml}), \mathrm{NBP} 1.6(20-40 \mu \mathrm{g} / \mathrm{ml})$, or $40 \mathrm{ng} / \mathrm{ml}$ of taprostene (i.e., a stable prostacyclin analog) (17). All aggregation studies were conducted in duplicate, and the results were expressed as a percentage of maximal aggregation ( $100 \%$ light transmittance).

Statistical analysis. All values in the text, table, and figures are presented as means \pm SEM of $n$ independent experiments. All data were subjected to ANOVA followed by the Bonferroni correction for posthoc $t$ test. $P \leq 0.05$ or less were considered to be statistically significant.

\section{Results}

Immunohistochemical localization of P-selectin. Coronary endothelial P-selectin was detected by using PB1.3 with a sensitive avidin-biotin immunoperoxidase procedure applied to 5$\mu \mathrm{m}$ thick plastic-embedded sections of heart tissue. The immunohistochemical results are illustrated in Fig. 1. Nonischemic (control) tissue demonstrated faint patchy cytoplasmic immunostaining primarily of the endothelial cells that lined coronary venules (Fig. $1 A$ ). Occasional weak cytoplasmic staining was demonstrated by endothelial cells lining epicardial arteries and veins. However, no cytoplasmic staining was observed on coronary capillary endothelium. Negative control immunohistochemical preparations, in which either the primary antibody (PB1.3; Fig. $1 B$ ) or the biotinylated secondary antibody (VectaStain Elite mouse IgG; Fig. $1 C$ ) was replaced by nonimmune serum, did not label endothelial cells. On the other hand, endothelial cell P-selectin was evident in ischemic-reperfused heart tissue when the nonblocking antibody NBP1.6 was applied $($ Fig. $1 D$ ) and when mAb PB1.3 was used (Fig. $1 E$ ). The peroxidase reaction product covered the full height of the cytoplasm rather than covering only the luminal surface of endothelial cells. The latter two figures show that the endothelial cell staining pattern for epicardial arteries was more intense and 

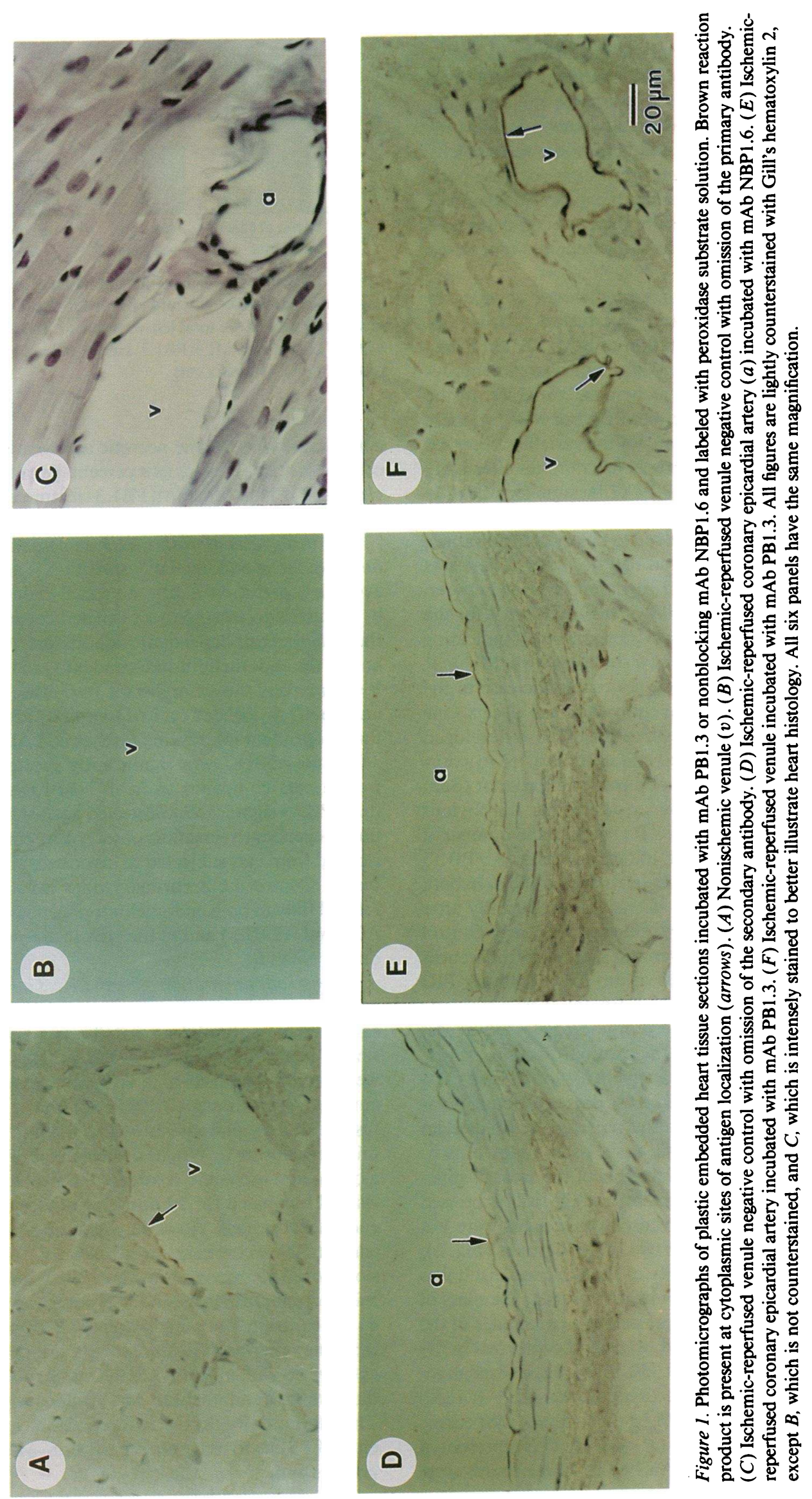
continuous than in nonischemic (control) heart tissue. The most intense and consistent cytoplasmic immunostaining was demonstrated by endothelial cells lining coronary venules of ischemic-reperfused heart tissue that was incubated with PB1.3 (Fig. $1 F$ ). Most of the venules had abundant cytoplasmic staining. P-selectin was also detected in arteries and arterioles, although the reaction product was usually patchy and less intense than for venules. Capillary endothelium showed a patchy distribution. Some capillaries were immunoperoxidase-positive while others were not. In other hearts exposed to $90 \mathrm{~min}$ of ischemia and $270 \mathrm{~min}$ of reperfusion, there was no detectable immunostaining in endothelial cells of the ischemic-reperfused coronary vasculature.

Cardiac electrophysiologic and hemodynamic changes. In the sham myocardial ischemia and reperfusion cats, we observed that intravenous administration of mAb PB1.3 (1 mg/ $\mathrm{kg}$ ) had no detectable effect on any of the measured hemodynamic, electrocardiographic, or biochemical variables. In the two groups of $\mathrm{MI}+\mathrm{R}$ cats given either PB1.3 or NBP1.6, there were no significant differences in any of the variables observed before coronary occlusion. However, a few minutes after LAD occlusion, the ST segment of the ECG became significantly elevated and peaked $60 \mathrm{~min}$ after coronary occlusion. After reperfusion, the ST segment decreased to nearly control values, indicating that effective reperfusion had occurred. There was no significant difference in peak ST segment elevation between the two $\mathrm{MI}+\mathrm{R}$ groups $(0.18 \pm 0.04$ vs. $0.19 \pm 0.02 \mathrm{mV}$ for the mAb NBP1.6 and mAb PB1.3 groups, respectively), indicating that the ischemic insult was similar in these two $\mathrm{MI}+\mathbf{R}$ groups. At reperfusion, there was a noticeable increase in the incidence of premature ventricular contractions in all cats. One cat in the MI + R group treated with mAb NBP1.6 developed ventricular fibrillation that was successfully converted to a normal sinus rhythm by using a DC electronic defibrillator (Sanborn Co, Waltham, MA). However, there was no obvious overall difference between the $\mathrm{MI}+\mathrm{R}$ groups in the number of PVCs occurring after reperfusion, indicating that $\mathrm{mAb}$ PB1.3 did not appear to exert any overt antiarrhythmic effect. In both groups of $\mathrm{MI}+\mathrm{R}$ cats, the PRI decreased significantly after coronary occlusion and gradually returned to nearly control values after reperfusion. There were no significant differences between the two $\mathrm{MI}+\mathrm{R}$ groups at any of the hourly PRI readings, suggesting that $\mathrm{mAb}$ PB1.3 did not exert a systemic hemodynamic effect (i.e., reduced myocardial oxygen demand), which could account for the cardioprotection.

Effect of $m A b P B 1.3$ on reperfusion myocardial injury. To ascertain the effects of mAb PB1.3 on the degree of actual myocardial salvage of ischemic tissue after reperfusion, we used an anatomic measurement of necrotic tissue as an index of ischemia reperfusion injury. The wet weights of the areas of myocardium subjected to ischemia (i.e., the area at risk) expressed as a percentage of the total left ventricular weights were not significantly different between the two MI + R groups (Fig. 2), further confirming that the severity of the ischemic insult was comparable in both ischemic groups. In contrast, the mass of the necrotic myocardial tissue expressed as a percentage of the mass of the myocardial area at risk was significantly less in the mAb PB1.3-treated group. About 35\% of the jeopardized myocardium developed into necrotic tissue in the $\mathrm{MI}+\mathrm{R} \mathrm{mAb}$ NBP1.6 group, whereas only $15 \%$ of the area at risk became necrotic in the mAb PB1.3 group $(P<0.01)$. This represents a $58 \%$ reduction in necrosis by $\mathrm{mAb} P B 1.3$. These results were

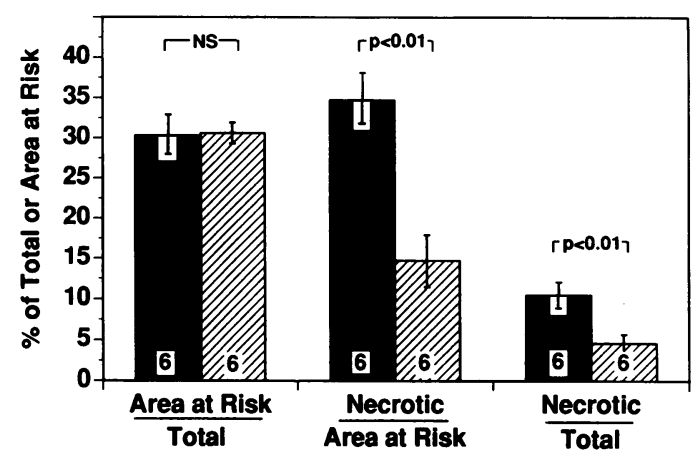

Figure 2. Tissue wet weight of area at risk as a percentage of the total left ventricular wet weight, and of necrotic tissue as a percentage of area at risk and of the total left ventricle for the two $M I+R$ groups. ๑, MI + NBP1.6; 四, MI + PB1.3. Height of bars are means; brackets represent \pm SEM for six cats.

comparable whether the necrotic area was expressed as a percentage of area at risk or as a percentage of the total left ventricle (Fig. 2). Thus, the mAb PB1.3 treatment resulted in significant attenuation of necrotic injury in the $\mathrm{MI}+\mathrm{R}$ cats.

In vitro effects of $\mathrm{MAb} P B 1.3$ on coronary endothelial dysfunction. Since endothelial dysfunction is an early and critical event in reperfusion injury, we tested endothelial dysfunction by comparing vasoactivity of isolated coronary artery rings to the endothelium-dependent vasodilators ACh and A-23187, and to the endothelium-independent vasodilator $\mathrm{NaNO}_{2}$. Fig. 3 summarizes the vasorelaxant responses to ACh, A-23187, and $\mathrm{NaNO}_{2}$ in isolated cat LAD coronary artery rings. Endothelium-dependent relaxation of ischemic LAD coronary rings to $\mathrm{ACh}$ and A23187 was significantly greater in mAb PB1.3treated cats compared to mAb NBP1.6-treated cats. Thus, mAb PB1.3 significantly protected against the loss of endothelium-dependent relaxation observed in coronary artery rings isolated from cats subjected to myocardial ischemia and reperfusion. Control LCX coronary rings isolated from all groups relaxed fully to both endothelium-dependent vasodilators (i.e., $\mathrm{ACh}$ and A23187) and to the endothelium-independent vasodilator $\mathrm{NaNO}_{2}$.

In vivo administration of $M A b P B 1.3$ inhibited $P M N$ adherence to ischemia reperfused coronary endothelium ex vivo. An early step in neutrophil-mediated injury is the increased adhesion of neutrophils to the vascular endothelium. In this study, we observed the effects of in vivo administered mAb PB1.3 on autologous unstimulated PMN adherence to ischemic-reperfused coronary endothelium (i.e., $90 \mathrm{~min}$ ischemia and 270 min reperfusion). When unstimulated PMNs were added alone to non-ischemic-reperfused control LCX coronary arteries and incubated for $20 \mathrm{~min}$, few neutrophils adhered to the endothelial surface. However, when unstimulated PMNs were added to LAD coronary arteries isolated $270 \mathrm{~min}$ after reperfusion from cats receiving mAb NBP1.6 and incubated for 20 min, a dramatic increase in PMN adherence was observed (Fig. 4). In contrast, when unstimulated PMNs were added to the ischemia reperfused LAD coronary arteries isolated from the cats treated with mAb PB1.3, the number of PMNs adhering to the coronary endothelium was significantly reduced (Fig. 4). Thus, the anti-P-selectin antibody significantly blocked adherence of PMNs to ischemia reperfusion-activated cat coronary endothelium. 

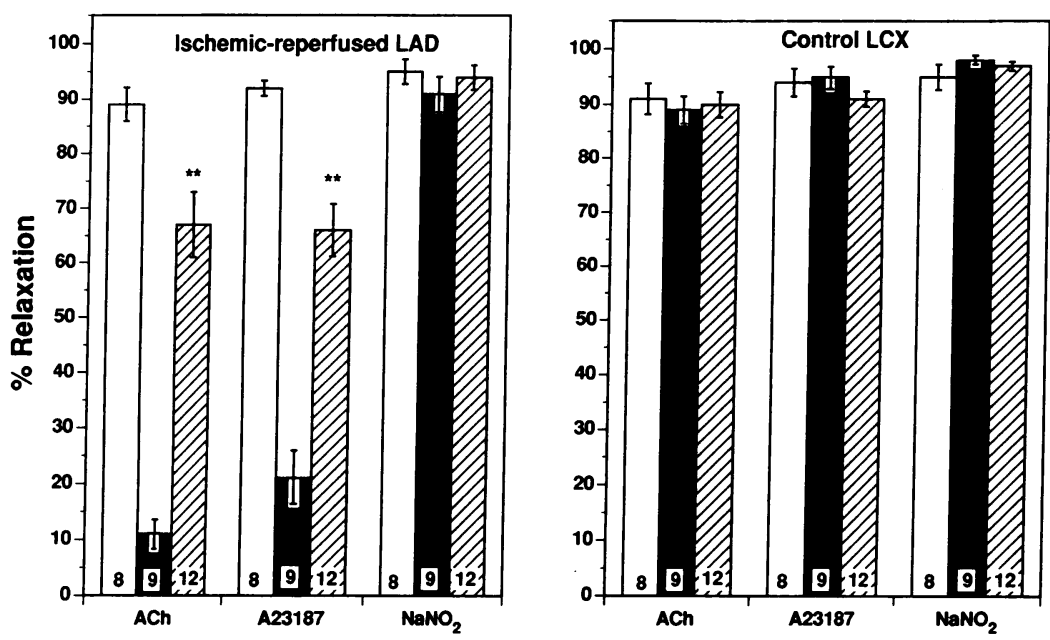

Figure 3. Summary of responses of ischemia reperfused LAD rings and nonischemic LCX coronary rings to $100 \mathrm{nM} \mathrm{ACh}, 1 \mu \mathrm{M} \mathrm{A} 23187$, and $100 \mu \mathrm{M}$ $\mathrm{NaNO}_{2}$. Bar heights are means; brackets indicate \pm SEM for 8-10 rings. $\square$, Sham MI + PB1.3; a, MI + NBP1.6; 0 , MI + PB1.3.

In vitro addition of $M A b P B 1.3$ on $P M N$ adherence to coronary endothelium in nonischemic control cats. To further examine the effect of mAb PB1.3 on PMN adherence to endothelial cells, we observed the effects of in vitro administration of $\mathrm{mAb}$ PB1.3 on PMN adherence to coronary artery endothelium. To upregulate P-selectin on the endothelial surface, we stimulated the coronary vascular endothelium with thrombin $(2 \mathrm{U} / \mathrm{ml})$ for $10 \mathrm{~min}$ (4). Addition of $\mathrm{mAb}$ PB 1.3 inhibited PMN adherence to thrombin stimulated endothelium in a concentration dependent manner (Fig. 5). At a concentration of $20 \mu \mathrm{g} / \mathrm{ml}$, a maximal inhibitory effect was obtained $(\sim 80 \%)$. Increasing the concentration of $\mathrm{MAb}$ PB1.3 did not attenuate PMN adherence any further. Fig. $6 \mathrm{~A}$ summarizes the results obtained from 10 to 13 coronary artery ring segments. Very few PMNs adhered to nonstimulated endothelium whereas large numbers of PMNs adhered to endothelium stimulated with thrombin. Addition of mAb PB1.3 significantly attenuated PMN adherence to the coronary endothelium while the nonblocking antibody (i.e., mAb NBP1.6) had no effect. Similar results were found when endothelial cells were incubated with histamine $(10 \mu \mathrm{M})$ for $10 \mathrm{~min}$, another agonist that upregulates P-selectin on the endothelial surface (4). Again, mAb PB1.3 significantly attenuated PMN adherence to histamine stimulated endothelial cells whereas mAb NBP1.6 had no effect (Fig. $6 B$ ).

Inhibitory effect of in vitro administration of $m A b P B 1.3$ on unstimulated PMN adherence to coronary artery endothelium after 90 min of ischemia and 20 min of reperfusion. The immunohistochemical results demonstrated that P-selectin was up-

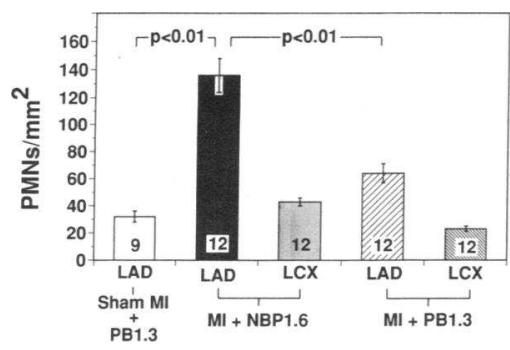

Figure 4. Effects of in vivo administration of $\mathrm{mAb}$ NBP1.6 and $\mathrm{mAb}$ PB1.3 on unstimulated neutrophil adherence to non-ischemic-reperfused LCX coronary endothelium and ischemic-reperfused LAD coronary endothelium ex vivo. Data are expressed as numbers of PMNs per square millimeter. Bar heights are means, brackets indicate \pm SEM, and numbers at the bottom of the bars are numbers of coronary rings studied. regulated in coronary vessel endothelial cells after $90 \mathrm{~min}$ of ischemia and $20 \mathrm{~min}$ of reperfusion. To determine whether P-selectin upregulation after $90 \mathrm{~min}$ of ischemia and $20 \mathrm{~min}$ of reperfusion plays an important role in PMN adherence to coronary artery endothelium, we examined the ability of $\mathrm{mAb}$ PB1.3 to attenuate this response in ischemic-reperfused coronary arteries. Fig. 7 summarizes the results of 7-10 coronary artery segments taken from three different cats. After 90 min of ischemia and 20 min of reperfusion, unstimulated PMN adherence to coronary artery endothelium was significantly increased, and this increased adhesiveness was significantly $(P$ $<0.01$ ) inhibited by $20 \mu \mathrm{g} / \mathrm{ml}$ of mAb PB1.3, the same concentration of mAb PB1.3 that inhibited PMN adherence to thrombin and histamine stimulated endothelial cells. In contrast, mAb NBP1.6 did not have any effect on PMN adherence to the ischemic-reperfused endothelial cells. These results confirm that P-selectin, expressed by endothelial cells after ischemia reperfusion, contributes significantly to the interaction between PMNs and endothelium.

Flow cytometric analysis of PB1.3 with cat platelets. The binding of mAb PB1.3 to freshly isolated cat platelets was confirmed by flow cytometry. Aliquots of cat platelets incubated with the control antibody immunoglobulin $G_{1}$ were only $4.6 \pm 0.2 \%$ positive with a mean channel fluorescence value of $2.3 \pm 0.1$. In contrast, addition of thrombin $(0.25 \mathrm{U} / \mathrm{ml})$ to cat platelets increased the percent of cells positive to PB1.3 to $30.1 \pm 2.0 \%$ with a mean channel fluorescence value of $7.2 \pm 0.3$. These values are 3.5-6 times that of control values. Binding of PB1.3 to nonthrombin activated platelets was minimal and was not significantly different from $\operatorname{IgG}_{1}$ controls. These results

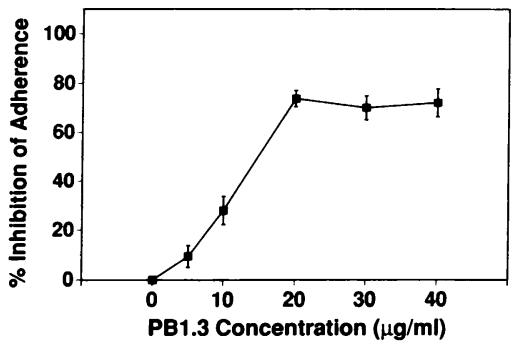

Figure 5. Concentration-inhibition response curve of $\mathrm{mAb}$ PB1.3 on unstimulated neutrophils to thrombin ( $2 \mathrm{U} /$ $\mathrm{ml}$ ) activated endothelial cells. Data are expressed as numbers of PMNs per square millimeter. Values are mean \pm SEM for six to eight coronary vascular strips. 

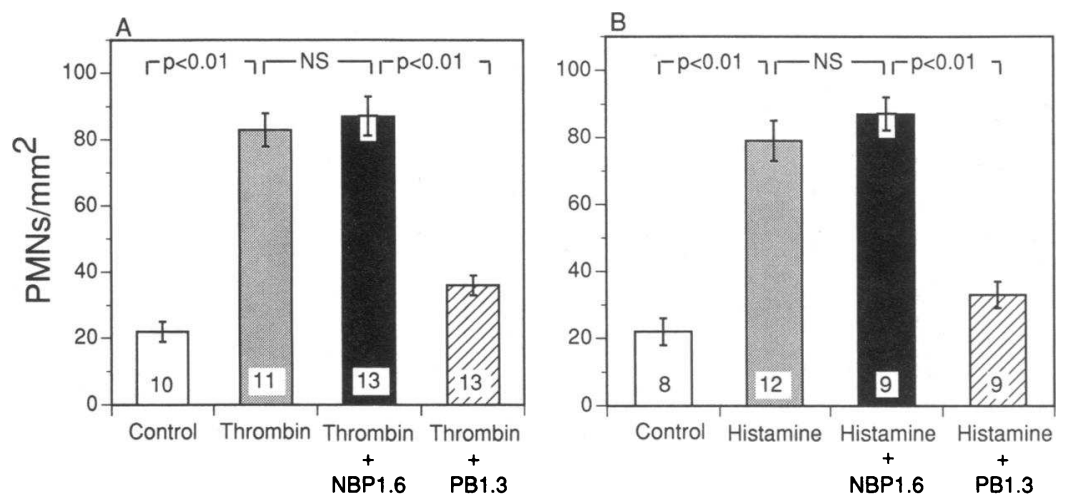

Figure 6. Effect of addition of mAb NBP1.6 (20 $\mu \mathrm{g} /$ $\mathrm{ml})$ and $\mathrm{mAb}$ PB1.3 $(20 \mu \mathrm{g} / \mathrm{ml})$ in vitro on coronary artery endothelium stimulated with $(A)$ thrombin $(2$ $\mathrm{U} / \mathrm{ml})$ or $(B)$ histamine $(10 \mu \mathrm{M})$. mAb NBP1.6 or mAb PB1.3 was added simultaneously with the PMNs and incubated for $20 \mathrm{~min}$. Data are expressed as numbers of PMNs $/ \mathrm{mm}^{2}$. Bar heights are means, brackets indicate \pm SEM, and numbers at the bottom of the bars are numbers of coronary rings studied. clearly demonstrate that $\mathrm{PB} 1.3$ reacts with thrombin activated cat platelets.

Platelet aggregation. Platelet aggregation to $0.1 \mathrm{U} / \mathrm{ml}$ of thrombin was $94 \pm 2 \%$ of maximum (i.e., $100 \%$ light transmittance) in washed cat platelets $(n=4)$. Addition of $40 \mu \mathrm{g} / \mathrm{ml}$ of either PB1.3 or NBP1.6 had no significant effect on cat platelet aggregation to thrombin. Specifically, PB1.3 inhibited thrombin induced aggregation by $2.3 \pm 2.1 \%$ (NS) and NBP1.6 inhibited aggregation by $1.1 \pm 1.5 \%$ (NS). In contrast, $40 \mathrm{ng} / \mathrm{ml}$ of taprostene, a prostacyclin analog with antiaggregatory activity, inhibited thrombin induced aggregation by $88 \pm 5 \%(P<0.01)$. Thus, PB1.3 does not appear to exert significant antiplatelet activity in feline platelets.

\section{Discussion}

P-selectin, also known as granule membrane protein-140, is one of three membrane glycoproteins in the selectin family of adhesion molecules that regulates interactions of leukocytes with the blood vessel wall (18). However, its potential role in pathophysiologic conditions is unclear since there have been very few in vivo studies that examine the role of P-selectin in

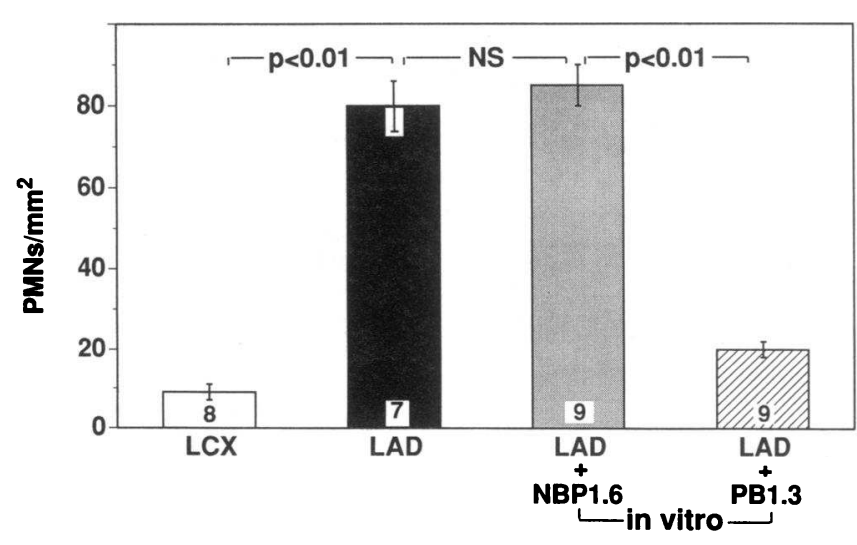

Figure 7. In vitro effects of mAb NBP1.6 $(20 \mu \mathrm{g} / \mathrm{ml})$ or mAb PB1.3 $(20 \mu \mathrm{g} / \mathrm{ml})$ on neutrophil adherence to LAD coronary arteries exposed to $90 \mathrm{~min}$ of ischemia and $20 \mathrm{~min}$ of reperfusion. Data are expressed as numbers of PMNs per square millimeter. Bar heights are mean values, brackets indicate \pm SEM values, and numbers at the bottom of the bars are the number of coronary strips studied. $L C X$, left circumflex coronary artery; $L A D$, left anterior descending coronary artery (ischemic-control reperfused). acute inflammation. The data obtained in this study provide striking evidence that intracellular expression of P-selectin in endothelial cells is markedly upregulated after $90 \mathrm{~min}$ of myocardial ischemia and $20 \mathrm{~min}$ of reperfusion in the cat. This substantiates earlier in vitro work demonstrating that P-selectin plays a significant role in PMN recruitment in inflammatory states $(4,11)$. Moreover, we found that a monoclonal antibody to P-selectin ( $\mathrm{mAb}$ PB1.3) preserves ischemic-reperfused myocardium by $\sim 60 \%$ compared to cats given a nonblocking antibody to P-selectin (mAb NBP1.6). Furthermore, the amount of ischemic myocardium that evolved into necrotic tissue in cats given mAb NBP1.6 was comparable to our most recent study that used $0.9 \% \mathrm{NaCl}$ as a vehicle control (19), indicating that mAb NBP1.6 did not have any detectable effect on infarct size.

It is unlikely that the cardioprotective actions of $\mathrm{mAb}$ PB1.3 were caused by differing degrees of ischemic injury between the two $\mathrm{MI}+\mathrm{R}$ groups since collateral flow to ischemic cat myocardium is only $5-7 \%$ of initial coronary flow $(15,20)$ and both ischemic groups exhibited comparable ST segment elevation and areas at risk of the left ventricle. Since $\mathrm{mAb}$ PB1.3 was administered for only the last 10 minutes of the 90-min ischemic period, decreases in ischemic severity because of a direct effect of mAb PB1.3 on collateral flow are also unlikely. In addition, mAb PB1.3 did not significantly alter the pressure rate index, and thus did not alter myocardial oxygen demand. PB1.3 was therefore comparable to recent studies from our laboratory, which used R15.7, an mAb against CD18, in $\mathrm{MI}+\mathrm{R}$ cats (15). Thus, one cannot attribute the cardioprotective effects of $\mathrm{mAb}$ PB1.3 to either alterations in myocardial oxygen supply or demand.

The myocardial salvage afforded by mAb PB1.3 is most likely caused by its anti-PMN effects in that it decreases PMN adhesion to ischemic-reperfused coronary endothelium. There is substantial evidence demonstrating that PMNs are one of the main contributors to myocardial injury associated with ischemia and reperfusion (21-23). In support of this, experiments designed to deplete neutrophils (21) have been shown to limit myocardial ischemia and reperfusion injury in the dog. Moreover, previous work from our laboratory (15) has demonstrated that a monoclonal antibody directed against CD18 of the neutrophil decreases myocardial necrosis and also attenuates endothelial dysfunction associated with reperfusion injury. Similarly, Perry and Granger (24) found that neutralization of CD18 on cat neutrophils significantly decreased neutrophil adherence and emigration in splanchnic ischemia and 
reperfusion. Thus, neutrophils play a significant role in myocardial ischemia and reperfusion, and inhibition of P-selectin should decrease neutrophil-endothelial interactions thereby attenuating myocardial necrosis.

The adhesive interaction between PMNs and the endothelium can be rapidly induced and is associated with a time dependent expression of adhesion molecules that target PMNs to the endothelial cell membrane (1). P-selectin, which is normally present in the Weibel-Palade bodies of endothelial cells, is rapidly translocated to the cell membrane upon stimulation with thrombin, histamine, or oxygen-derived free radicals, where it tethers PMNs to the endothelial cell surface $(4,11)$. Since ischemia reperfusion results in the elaboration of a variety of humoral mediators, P-selectin should be expressed within minutes after the onset of reperfusion. Our results provide direct evidence that $P$-selectin is expressed intracellularly within arterial and venular endothelial cells within $20 \mathrm{~min}$ of reperfusion. Because our immunohistochemical method revealed cytoplasmic staining, we were unable to distinguish surface expression of P-selectin. However, at $270 \mathrm{~min}$ after reperfusion, we did not observe any immunohistochemical evidence of P-selectin expression in coronary endothelial cells, consistent with Palluy et al. (25), who found that endothelial cells exposed to hypoxia and reoxygenation expressed P-selectin after $20 \mathrm{~min}$ but not $240 \mathrm{~min}$ of reoxygenation. Therefore, our results suggest that P-selectin expression on the endothelial cell surface was early and transient after ischemia and reperfusion. Furthermore, we demonstrated that coronary artery endothelium stimulated by ischemia reperfusion, thrombin, or histamine exhibited comparable increases in PMN adherence, a process which mAb PB1.3 significantly inhibited. Based on these results, one can conclude that P-selectin is an important adhesion molecule regulating PMN adhesion to ischemic-reperfused endothelium.

The PMN ligand to P-selectin is not known with certainty, but some recent evidence suggests that it is an L-selectin associated oligosaccharide, specifically sialyl Lewis ${ }^{x}(26,27)$. However, other evidence points to a $\mathrm{Ca}^{2+}$ dependent ligand for P-selectin that is distinctly different from L-selectin (28). Although the exact ligand for P-selectin remains to be elucidated, P-selectin undoubtedly plays a significant role in trafficking of PMNs to the endothelial cell surface.

Targeting of PMNs to the endothelial cell surface allows PMNs to be activated, thereby shedding L-selectin and upregulating CD11/CD18 on the PMN plasma membrane (29). One humoral mediator, platelet activating factor (PAF), is known to increase following ischemia and reperfusion, and facilitates the shedding of L-selectin via its receptor on neutrophils (19, 30). Recent observations by Patel et al. (31) have demonstrated that oxidant injury to the endothelium also stimulates neutrophils through a PAF receptor except that an oxidized phospholipid, rather than native PAF, may be the relevant molecular species in this case. Whatever the precise mechanism of neutrophil activation, the current report on mAb PB1.3 and earlier data using WEB 2170, a specific PAF receptor antagonist, indicate that both agents reduce myocardial necrosis and PMN adhesion to endothelial cells to similar degrees after myocardial ischemia and reperfusion (19).

After activation with chemotactic factors such as PAF or PAF-like phospholipids, PMNs shed L-selectin and adhere tightly to endothelial cells. This adhesive interaction is mediated by $\mathrm{CD} 11 / \mathrm{CD} 18$ adhesion glycoproteins located on the neutrophil plasma membrane. These $\beta_{2}$ integrins are functionally upregulated upon PMN activation and recognize intercellular adhesion molecules 1 and 2 (ICAM-1 and ICAM-2) of the immunoglobulin supergene family (32). Binding of CD11/ CD1 8 integrins on activated PMNs to ICAM- 1 facilitates transendothelial migration and intensifies neutrophil-mediated injury to ischemic-reperfused myocardium (33). In this connection, we have previously demonstrated that monoclonal antibodies to either CD18 on the PMN or to ICAM-1 on the endothelial cell significantly inhibit PMN adherence and protect myocardial tissue from reperfusion injury $(15,34)$. Thus, if PMNs become activated, significant adhesion to the endothelial surface occurs, which contributes significantly to reperfusion-induced myocardial injury. Presumably, P-selectin upregulation on the endothelial cell surface is a prerequisite for maximal interaction between CD11/CD18 and ICAM-1. Therefore, it is possible that $\mathrm{mAb} P B 1.3$ prevents initial targeting of $\mathrm{PMNs}$ to the endothelial cell surface, thereby reducing tight adhesive interaction caused by CD11/CD18-ICAM mechanisms.

Trafficking of PMNs to the endothelial cell surface by P-selectin may also result in PMN mediated endothelial injury. Upon activation, PMNs induce significant endothelial dysfunction that is characterized by reduced release of both agonist stimulated and basally released nitric oxide (NO) from endothelial cells $(7,8)$. These effects appear to be mediated by superoxide radicals generated by activated PMNs that either inactivate or decrease the synthesis of $\mathrm{NO}$ (35). Several studies $(9,36)$ have demonstrated that $N O$ reduces $P M N$ adherence to endothelial cells, suggesting that NO is an important intrinsic modulator of leukocyte adherence. Moreover, NO release is significantly diminished after ischemia and reperfusion and its decreased release is associated with significant myocardial injury (7-8, 37-38). Conversely, if NO release is preserved after reperfusion, cardioprotection occurs characterized by significant reductions in both myocardial tissue injury and PMN adherence to the ischemic-reperfused endothelium (36). Although no data are currently available, NO may act to attenuate upregulation of P-selectin on the surface of the endothelium. This inhibitory influence could dissipate upon reperfusion when endothelial dysfunction occurs.

In this study, endothelial dysfunction and PMN adherence to the arterial endothelium were significantly attenuated after $90 \mathrm{~min}$ of ischemia and $270 \mathrm{~min}$ of reperfusion in cats treated with mAb PB1.3 compared to mAb NBP1.6. Thus, mAb $\mathrm{PB} 1.3$ prevents extensive endothelial dysfunction that is normally associated with reperfusion injury, thereby sustaining NO release that may act to retard PMNs from adhering to the endothelial cell surface. Alternatively, sustained P-selectin expression has been demonstrated in endothelial cells exposed to $\mathrm{H}_{2} \mathrm{O}_{2}$ allowing $\mathrm{P}$-selectin to act alone in promoting PMN adhesion (11) and mAb PB1.3 may inhibit this interaction during the entire reperfusion period. Whatever the precise molecular mechanism, mAb PB1.3 reduces PMN adherence to the ischemic-reperfused endothelium, thereby preserving endothelial function. Reductions in this adhesive interaction may attenuate the release of PMN-derived cytotoxic substances that play an important role in cardiac myocyte injury.

One can not rule out the possibility that PB1.3 affords cardioprotection by binding to cat platelets and thereby inhibiting platelet interaction with other cells. However, Engler et al. (39) previously found no histologic evidence of platelet plugging in reperfused myocardium. Other studies have observed no re- 
duction in infarct size after marked platelet depletion (40-42). In support of this, we found that PB1.3 does not inhibit platelet aggregation induced by thrombin. Platelets, however, can interact with neutrophils via P-selectin expressed on plasma membranes of platelets after activation. PB1.3 blocks this interaction in human platelets and neutrophils (12), and it may also block cat platelet and neutrophil interaction. In vitro studies have shown that substances released from platelets augment leukocyte adhesiveness (42-44). A more recent study, however, found that rolling of leukocytes in vivo is inhibited by activated platelets or damaged vascular cells (45). Thus, the exact role of platelets in augmenting PMN effects in settings of myocardial ischemia and reperfusion is not known with certainty, but it is unlikely to play a major role in this model of reperfusion injury.

In conclusion, we have demonstrated that significant upregulation of P-selectin occurs in ischemic-reperfused endothelial cells and this increased expression is associated with significant PMN adherence to the endothelium. Moreover, administration of a monoclonal antibody to P-selectin just before reperfusion significantly attenuated myocardial injury and endothelial dysfunction. To our knowledge, this is the first in vivo study examining the role of $\mathrm{P}$-selectin in ischemia reperfusion injury. These results confirm that PMNs mediate myocardial tissue injury after reperfusion, and in particular, demonstrate that P-selectin plays an important role in modulating PMN-endothelial interactions associated with ischemia reperfusion injury.

\section{Acknowledgments}

The authors thank Drs. Margaret Polley, Les Walker, and Michael Forrest of Cytel Inc., for the supply of mAb PB1.3 and mAb NBP1.6 and Xiao-yin Li for her help with neutrophil isolation and labeling. We also gratefully acknowledge David Rosolia for his excellent technical assistance in immunohistochemical localization of P-selectin. The authors would also like to thank Dr. Bruce Bochner and Sherry Sterbinsky for their assistance on flow cytometry studies.

This work was supported by research grant no. GM-45434 from the National Institute of General Medical Science of the National Institutes of Health. A. S. Weyrich is a National Institutes of Health postdoctoral fellow (HL-08057). Xin-liang $\mathrm{Ma}$ is a senior research fellow of the Ischemia-Shock Research Institute.

\section{References}

1. Zimmerman, G. A., S. M. Prescott, and T. M. McIntyre. 1992. Endothelial cell interactions with granulocytes: tethering and signaling molecules. Immunol. Today. 13:93-99.

2. McEver, R. P. 1991. Properties of GMP-140, an inducible granule membrane protein of platelets and endothelium. J. Cell Biochem. 45:156-161.

3. McEver, R. P., J. H. Beckstead, K. L. Moore, L. Marshall-Carlson, and D. F. Bainton. 1989. GMP-140, a platelet $\alpha$-granule membrane protein, is also synthesized by vascular endothelial cells and is localized in Weibel-Palade bodies. J. Clin. Invest. 84:92-99.

4. Lorant, D. E., K. D. Patel, T. M. McIntyre, R. P. McEver, S. M. Prescott, and G. A. Zimmerman. 1991. Coexpression of GMP-140 and PAF by endothelium stimulated by histamine or thrombin: a juxtacrine system for adhesion and activation of neutrophils. J. Cell Biol. 115:223-234.

5. Harlan, J. M. 1985. Leukocyte-endothelial interactions. Blood. 65:513525.

6. Mullane, K. M. 1991. Neutrophil and endothelial changes in reperfusion injury. Trends Cardiovasc. Med. 1:282-289.

7. Tsao, P. S., N. Aoki, D. J. Lefer, G. Johnson III, and A. M. Lefer. 1990. Time course of endothelial dysfunction and myocardial injury during myocardial ischemia and reperfusion in the cat. Circulation. 82:1402-1412.
8. Ma, X-I, A. S. Weyrich, D. J. Lefer, and A. M. Lefer. 1993. Diminished basal nitric oxide release after myocardial ischemia and reperfusion promotes neutrophil adherence to coronary endothelium. Circ. Res. 72:403-412.

9. Kubes, P., M. Suzuki, and D. N. Granger. 1991. Nitric oxide: an endogenous modulator of leukocyte adhesion. Proc. Natl. Acad. Sci. USA. 88:46514655 .

10. Tsao, P. S., and A. M. Lefer. 1990. Time course and mechanism of endothelial dysfunction in isolated ischemic- and hypoxic-perfused rat hearts. Am. J. Physiol. 259:H1660-H1666.

11. Patel, K. D., G. A. Zimmerman, S. M. Prescott, R. P. McEver, and T. M. McIntyre. 1991. Oxygen radicals induce human endothelial cells to express GMP-140 and bind neutrophils. J. Cell Biol. 112:749-759.

12. Mulligan, M. S., M. J. Polley, R. J. Bayer, M. F. Nunn, J. C. Paulson, and P. A. Ward. 1992. Neutrophil-dependent acute lung injury: requirement for P-selectin (GMP-140). J. Clin. Invest. 91:577-587.

13. Lafredo, L. J., and R. G. Olsen. 1986. Demonstration of depressed polymorphonuclear leukocyte function in non-viremic FeLV-infected cats. Cancer Invest. 4:297-300.

14. Yuan, Y., and B. P. Fleming. 1990. A method for isolation and fluorescent labeling of rat neutrophils for intravital microvascular studies. Microvasc. Res. 40:218-229.

15. Ma, X-I, P. S. Tsao, and A. M. Lefer. 1991. Antibody to CD-18 exerts endothelial and cardioprotective effects in myocardial ischemia and reperfusion. J. Clin. Invest. 88:1237-1243.

16. Beckstead, J. H., P. E. Stenberg, R. P. McEver, M. A. Shuman, and D. F. Bainton. 1986. Immunohistochemical localization of membrane and $\alpha$-granule proteins in human megakaryocytes: application to plastic-embedded bone marrow biopsy specimens. Blood. 67:285-293.

17. Lefer, A. M., and H. Darius. 1989. Taprostene (CG-4203). Cardiovasc. Drug Rev. 7:39-51.

18. McEver, R. P. 1991. Leukocyte interactions mediated by selectins. Thromb. Haemostasis. 65:223-228.

19. Ma, X-I, A. S. Weyrich, S. Krantz, and A. M. Lefer. 1992. Mechanisms of the cardioprotective action of WEB-2170, a platelet activating factor antagonist, in myocardial ischemia and reperfusion. J. Pharmacol. Exp. Therap. 260:12291236.

20. Westby, J., E. Hexeberg, J. Olweus, O. L. Myking, J. Lekven, and K. Grong. 1991. Blood flow regulation during acute regional ischemia in feline hearts: importance of postjunctional $\alpha_{1}$ - and $\alpha_{2}$-adrenoceptors. J. Cardiovasc. Pharmacol. 18:487-495.

21. Litt M. R., R. W. Jeremy, H. F. Weisman, J. A. Winkelstein, and L. C. Becker. 1989. Neutrophil depletion limited to reperfusion reduces myocardial infarct size after 90 minutes of ischemia: evidence for neutrophil-mediated reperfusion injury. Circulation. 80:1816-1827.

22. Entman, M. L., L. Michael, R. D. Rossen, W. J. Dreyer, D. C. Anderson, A. A. Taylor, and C. W. Smith. 1991. Inflammation in the course of early myocardial ischemia. FASEB (Fed. Am. Soc. Exp. Biol.) J. 5:2529-2537.

23. Kloner, R. A., F. Giacomelli, K. J. Alker, S. L. Hale, R. Matthews, and S. Bellows. 1991. Influx of neutrophils into the walls of large epicardial coronary arteries in response to ischemia/reperfusion. Circulation. 84:1758-1772.

24. Perry, M. A., and D. N. Granger. 1992. Leukocyte adhesion in local versus hemorrhage-induced ischemia. Am. J. Physiol. 263:H810-H815.

25. Palluy, O., L. Morliere, J. C. Gris, C. Bonne, and G. Modat. 1992. Hypoxia/reoxygenation stimulates endothelium to promote neutrophil adhesion. Free Radical Biol. \& Med. 13:21-30.

26. Polley, M. J., M. L. Phillips, E. Warner, E. Nudelman, A. K. Singal, S. I Hakomori, and J. C. Paulson. 1991. CD62 and endothelial cell leukocyte adhesion molecule (ELAM-1) recognize the same carbohydrate ligand, sialyl Lewis X. Proc. Soc. Natl. Acad. Sci. USA. 88:6224-6228.

27. Picker, L. J., R. A. Warnock, A. R. Burns, C. M. Doerschuk, E. L. Berg, and E. C. Butcher. 1991. The neutrophil selectin LECAM-1 presents carbohydrate ligands to the vascular selectins ELAM-1 and GMP-140. Cell. 66:921-933.

28. Moore, K. L., N. L. Stults, S. Diaz, D. F. Smith, R. D. Cummings, A. Varki, and R. P. McEver. 1992. Identification of a specific glycoprotein ligand for P-selectin (CD62) on Myeloid Cells. J. Cell Biol. 118:445-456.

29. Von Andrian, U. H., J. D. Chambers, L. M. McEvoy, R. F. Bargatze, K-E. Arfors, and E. C. Butcher. 1991. Two-step model of leukocyte-endothelial cell interaction in inflammation: distinct roles for LECAM-1 and the leukocyte $\beta_{2}$ integrins in vivo. Proc. Natl. Acad. Sci. USA. 88:7538-7542.

30. Ko, W., A. S. Hawes, W. D. Lazenby, S. E. Calvano, Y. T. Shin, J. A. Zelano, A. C. Antonacci, O. W. Isom, and K. H. Krieger. 1991. Platelet activating factor stimulates polymorphonuclear leukocyte hydrogen peroxide production during myocardial reperfusion. J. Thorac. Cardiovasc. Surg. 102:297-308.

31. Patel, K. D., G. A. Zimmerman, S. M. Prescott, and T. M. McIntyre 1992. Novel leukocyte agonists are released by endothelial cells exposed to peroxide. J. Biol. Chem. 267:15168-15175.

32. Smith, C. W., R. Rothlein, B. J. Hughes, M. M. Mariscalco, H. E. Rudloff, F. C. Schmalstieg, and D. C. Anderson. 1988. Recognition of an endothelial determinant for CD18-dependent human neutrophil adherence and transendothelial migration. J. Clin. Invest. 82:1746-1756. 
33. Smith, C. W., S. D. Marlin, R. Rothlein, C. Toman, and D. C. Anderson. 1989. Cooperative interaction of LFA-1 and Mac-1 with intercellular adhesion molecule-1 in facilitating adherence and transendothelial migration of human neutrophils in vivo. J. Clin. Invest. 83:2008-2017.

34. Ma, X-I, D. J. Lefer, A. M. Lefer, and R. Rothlein. 1992. Coronary endothelial and cardiac protective effects of a monoclonal antibody to intercellular adhesion molecule-1 in myocardial ischemia and reperfusion. Circulation. 86:937-946.

35. Ma, X-I, P. S. Tsao, G. E. Viehman, and A. M. Lefer. 1991. Neutrophilmediated vasoconstriction and endothelial dysfunction in low-flow perfusion-reperfused cat coronary artery. Circ. Res. 69:95-106.

36. Weyrich, A. S., X-1 Ma, and A. M. Lefer. 1992. The role of L-arginine in ameliorating reperfusion injury after myocardial ischemia in the cat. Circulation. $86: 279-288$

37. Van Benthuysen, K. M., I. F. McMurtry, and L. D. Horwitz. 1987. Reperfusion after acute coronary occlusion in dogs impairs endothelium-dependent relaxation to acetylcholine and augments contractile reactivity in vitro. J. Clin. Invest. 79:265-274.

38. Mehta, J. L., D. L. Lawson, and W. W. Nichols. 1989. Attenuated coronary relaxation after reperfusion: effects of superoxide dismutase and $\mathrm{TxA}_{2}$ inhibitor U-63557. Am. J. Physiol. 257:H1240-H1246.
39. Engler, R., G. W. Schmid-Schonbein, and R. S. Pavelec. 1983. Leukocyte capillary plugging in myocardial ischemia and reperfusion in the dog. Am. J. Path. 111:98-111.

40. Jolly, S. R., W. A. Schumacher, S. L. Kunkel, G. D. Abrams, J. Liddicoat, and B. R. Lucchesi. 1985. Platelet depletion in experimental myocardial infarction. Basic Res. Cardiol. 80:269-279.

41. Mullane, K. M., and J. C. McGiff. 1985. Platelet depletion and infarct size in an occlusion-reperfusion model of myocardial ischemia in anesthetized dogs. J. Cardiovasc. Pharmacol. 7:733-738.

42. Turner, S. R. J. A. Tainer, and W. S. Lynn. 1975. Biogenesis of chemotactic molecules by the arachidonate lipoxygenase system of platelets. Nature (Lond.). 257:680-681.

43. Boogaerts, M. A., O. Yamada, H. S. Jacob, and C. F. Moldow. 1982 Enhancement of granulocyte-endothelial cell adherence and granulocyte-induced cytotoxicity by platelet released products. Proc. Natl. Acad. Sci. USA. 79:7019-7023.

44. Lucchesi, B. R., J. K. Mickelson, J. W. Homeister, and C. V. Jackson. 1987. Interaction of the formed elements of blood with the coronary vasculature in vivo. Fed. Proc. 46:63-72.

45. Oude Egbrink, M. G. A., G-J. Tangelder, D. W. Slaaf, and R. S. Reneman. 1992. Influence of platelet-vessel wall interactions on leukocyte rolling in vivo. Circ. Res. 70:355-363. 